\title{
Network Pharmacology-based Strategy for Predicting Active Ingredients and Potential Targets of Coptis Chinensis Franch in Polycystic Ovary Syndrome
}

\section{Jianxiong Ma}

Zhejiang Chinese Medical University

\section{Miaoyong Ye}

Zhejiang Chinese Medical University

\section{$\mathrm{Ke} \mathrm{Ma}$}

Zhejiang Chinese Medical University

\section{Kang Zhou}

Zhejiang Chinese Medical University

\section{YingYing Zhang}

Zhejiang Chinese Medical University

\section{Xiting Wang}

Beijing University of Chinese Medicine

\section{Hongxuan Tong ( $\nabla$ thongxuan1@163.com )}

Institute of Basic Theory for Chinese Medicine, China Academy of Chinese Medical Sciences, Beijing, China

\section{Research}

Keywords: Coptis chinensis, molecular docking, network pharmacology, polycystic ovary syndrome, AGEs/RAGE signaling pathways, IL-1B signaling pathways, IL-17 signaling pathways, MAPK signaling pathways, CXCL8 signaling pathways

Posted Date: September 17th, 2020

DOI: https://doi.org/10.21203/rs.3.rs-77454/v1

License: (c) (i) This work is licensed under a Creative Commons Attribution 4.0 International License. Read Full License 


\section{Abstract}

Background: Polycystic ovary syndrome (PCOS) is a disease that causes low fertility in females. Coptis chinensis (CC) has been used to clear away heat and dampness, purify fire, and detoxify in traditional Chinese medicine (TCM). Although CC has demonstrated efficacy against PCOS in clinical practice, there is no available data regarding the bioactive ingredients, component targets, and confirmed molecular mechanism of this drug combination.

Methods: A network pharmacology approach was applied to analyze the bioactive ingredients, component targets, and core signaling pathways of CC. The TCM systems pharmacology database and analysis platform (TCMSP) was used to screen effective active ingredients and targets of CC. The GeneCards, OMIM, and PharmGkb databases was utilized to screen disease targets for PCOS. R language software was used to screen common targets of drugs and diseases. Cytoscape software (version 3.7.1) was utilized to build a drug-active ingredient-disease target interaction network, and the STRING platform was utilized to construct a common target protein-protein interaction network, including 102 nodes and 221 edges. OmicShare tools was used to analysis Gene ontology (GO) biological function analysis and Kyoto Encyclopedia of Genes and Genomes (KEGG) path enrichment. Schrodinger software was used to evaluate the interaction between active components and their targets and explore binding modes.

Results: 14 effective active ingredients and 218 targets of CC were screened by TCMSP platform. 3517 disease targets for PCOS were obtianed by the disease database and 102 common targets of drugs and diseases were screened through $\mathrm{R}$ language software. Key targets of $\mathrm{CC}$ for the treatment of PCOS included JUN, MAPK, IL-6, CXCL8, FOS, and IL1B. A total of 123 Gene Ontology (GO) terms and 129 pathways were acquired by analyzing the enrichment of GO and KEGG. It is speculated that the AGEs/RAGE, TNF, IL-17, MAPK and HIF-1 signaling pathways are closely related to PCOS and may be the core pathways involved in PCOS. The molecular docking results showed that quercetin has a higher degree of binding to core targets (eg: IL-6, IL-1 $\beta$, MAPK, CXCL8) related to the inflammatory pathway.

Conclusions: This preliminary study verified the basic pharmacological effects and mechanisms of CC, a Chinese medicine, in the treatment of PCOS. Particularly, the effect of CC on inflammation and glucose metabolism pathway was noteworthy. This study provides new insights for the systematic exploration of the mechanism of action of Chinese medicine.

\section{Background}

Polycystic ovarian syndrome (PCOS) is an endocrine syndrome characterized by persistent anovulation, hyperandrogenemia or insulin resistance, and is the most common cause of infertility in women of childbearing age [1]. In recent years, PCOS has been defined as a metabolic syndrome because it is often accompanied by metabolic disorders such as obesity, insulin resistance, hyperlipidemia, and long-term risk of type 2 diabetes, hypertension, and other cardiovascular diseases, seriously affecting the physical and mental health of women of childbearing age [2,3]. At present, the treatment for insulin resistance in 
PCOS patients is mainly to increase insulin sensitivity in peripheral tissues, and the representative drug is metformin. Although metformin can obtain a certain effect temporarily, it has many side effects, it cannot be used for a long time, and the recurrence rate after stopping the drug is high [4]. Therefore, it is important to find a PCOS replacement or adjuvant therapy with less side effects and effective results.

In China, Coptis chinensis (CC) is a commonly used botanical medicine with a long history and is widely used to treat various diseases, including gynecological diseases and diabetes. In the theory of Chinese medicine, CC can clear away heat and dampness, purify fire, and detoxify the body. Pharmacological studies have confirmed that CC has anti-inflammatory, anti-allergenic, hypoglycemic, lipid-lowering, and anti-oxidant properties [5, 6]. Of note, it can improve insulin resistance and impaired glucose tolerance in PCOS patients $[7,8]$. However, based on the multi-target and multi-path pharmacological characteristics of Chinese herbal medicines, the current research of CC in the treatment of PCOS still has shortcomings such as low sensitivity, single reliable evaluation index, and inability to systematically evaluate it. It is difficult to make a scientific, effective, and comprehensive explanation of its mechanism of action. Therefore, it is important to systematically and comprehensively study the material basis and mechanisms of action of $\mathrm{CC}$ to improve and treat PCOS.

Network pharmacology is based on the theory of systems biology. A model is established for predicting the relationship between drugs and targets, diseases and therapeutic targets, integrating the network of interactions between them, and analyzing the interaction of active ingredients and targets in the network module. It is a systematic and holistic approach to the study of the relationships between active pharmaceutical ingredients and potential targets $[9,10]$. It can scientifically and comprehensively evaluate the potential active ingredients, targets, and mechanisms of action of traditional Chinese medicine (TCM) and traditional Chinese medicinal compounds [11].

In this study, a network pharmacology method was used to analyze the active ingredients of a TCM (CC) and explore its possible targets and mechanisms of action in treating PCOS, in order to clarify its scientific connotations, improve its mechanisms of action, and apply Chinese medicine or adjuvant therapy in clinical situations (Fig. 1).

\section{Methods}

\section{Research material}

The following databases and resources were used: Chinese Herbal Medicine System Pharmacology Platform (TCMSP) database (http://Isp.nwu.edu.cn/tcmsp.php); Uniprot database (http://www.uniprot.org); GeneCards database (https://www. genecards.org/), OMIM database (https://www.omim.org/), PharmGkb database (https://www.pharmgkb.org/); functional protein contact network (STRING) database (https://string -db.org/); Cytoscape 3.7.1 (https://cytoscape.org/); R language software Rx64 3.6.2 (https://www.r-project.org/); Bioconductor 
(https://www.bioconductor.org/), an R plug in source; and Microsoft Windows 10 Home operating environment.

\section{Collection of bioactive compounds of Coptis chinensis}

TCMSP, a traditional Chinese medicine (TCM) ingredients database, is commonly used for researching Chinese medicine network pharmacology. It contains more than 500 herbal medicines and more than 3,000 kinds of Chinese herbal medicines. It has collected more than 3300 targets of ingredients from databases such as DrugBank and HIT. TCMSP was used to search all the active ingredients in the traditional Chinese medicine, CC. According to the research results, screening was performed with oral bioavailability $(O B) \geq 30 \%$ and drug-likeness $(D L) \geq 0.18$ as the limiting conditions to obtain the biologically active components of CC.

\section{Prediction of target of Coptis chinensis}

Targets of effective active ingredients of CC were collected from the TCMSP database. For the convenience and standardization of data processing, the Uniprot database was used to uniformly convert the "protein name" of the target into the "gene name".

\section{Disease targets for polycystic ovary syndrome}

Using the search term "Polycystic Ovary Syndrome", the databases GeneCards, OMIM, and PharmGkb were interrogated. These platforms are comprehensive disease-related data platforms, and there are a wide range of data related to complex diseases, including data literature, and experimental verifications. Search results from the three platforms were refined to obtain the disease targets of PCOS.

\section{Screening of common targets for drugs and diseases and construction of interaction networks}

R software was used to obtain the common target of drugs and diseases, and a Venn diagram was drawn. The STRING platform was used to construct the common target protein-protein interaction (PPI). The minimum interaction threshold was set to "highest confidence" (>0.9). "Node" was used to represent different targets, and "edge" represented the relationship between different targets. $\mathrm{R}$ software was used to count the frequency of occurrence and to produce a histogram. All R packages were downloaded via the $\mathrm{R}$ and Bioconductor websites.

\section{Construction of drug-active ingredient-disease-target interaction network}

Cytoscape 3.7.1 (https://cytoscape.org/) software was used to build a drug-active ingredient-diseasetarget interaction network. In the resulting network diagram, the software defines nodes and edges. "Node" represents drugs, active ingredients, diseases, and targets, and "edge" represents the relationship between the above nodes. 
Gene ontology (GO) biological function analysis and Kyoto Encyclopedia of Genes and Genomes (KEGG) path enrichment analysis

GO and KEGG data were analyzed using OmicShare tools (www.omicshare.com/tools), and the results were presented as bubble charts. $P$ was defined as significant at a value $<0.05$. The degree of enrichment of core pathways was analyzed based on the value of the enrichment factor to explore the possible mechanism of CC in treating PCOS. In addition, the ClueGo plug-in in Cytoscape 3.7.1 software was used to perform GO analysis on common targets of drugs and diseases, and the results were presented as an interaction network and a pie chart.

\section{Molecular docking}

The interaction between active components and their targets was evaluated to explore the binding modes; four components and four targets were chosen. PubChem (https://pubchem.ncbi.nlm.nih.gov/) was used to collect the 3D structure data of quercetin (CID 5280343), berberrubine (CID 72703), berberine (CID 2353), and canadine (CID 34458). PDB data were used for the structures of MAPK1 (PDBID: 6G54), CXCL8 (PDBID: 5D14), IL-6 (PDBID: 409H), and IL-1 $\beta$ (PDBID: 4G6M). Schrodinger software (v9.2; https://www.schrodinger.com/) was used to pretreat compound structures and generate multiple conformations. Protein structures were processed by removing hydrone and adding hydrogen atoms, and the Sitmap module was used to explore and define binding sites. The liganddock module was used to simulate the molecular docking of compounds and proteins, and the docking score was evaluated using the Docking score function. Simultaneously, the visual analysis module was used to observe the binding postures of molecules and their targets.

\section{Results}

\section{Screening of active components of Coptis chinensis}

The TCMSP database contained 48 species of CC. According to the screening conditions, there were 26 components with $\mathrm{OB} \geq 30 \%, 31$ kinds with $\mathrm{DL} \geq 0.18$, and 14 kinds of effective serum active ingredients with $\mathrm{OB} \geq 30 \%$ and $\mathrm{DL} \geq 0.18$. These included berberine, obacunone, berberrubine, epiberberine, canadine, berlambine, corchoroside A, magnograndiolide, palmidin A, palmatine, quercetin, coptisine, worenine, and moupinamide (Table 1). 
Table 1

Active ingredients of Coptis chinensis selected from TCMSP

\begin{tabular}{|lllll|}
\hline Molecular number & Active ingredients & Chemical formula & OB \% & DL \\
\hline MOL001454 & berberine & C2OH18CINO4 & 36.86 & 0.19 \\
\hline MOL013352 & obacunone & C26H3007 & 43.29 & 0.31 \\
\hline MOL002894 & berberrubine & C19H16CINO4 & 35.74 & 0.24 \\
\hline MOL002897 & epiberberine & C20H17NO5 & 43.09 & 0.19 \\
\hline MOL002903 & (R)-Canadine & C2OH21NO4 & 55.37 & 0.2 \\
\hline MOL002904 & berlambine & C20H17NO5 & 36.68 & 0.28 \\
\hline MOL002907 & corchoroside A & C29H42O9 & 104.95 & 0.29 \\
\hline MOL000622 & magnograndiolide & C15H22O4 & 63.71 & 0.3 \\
\hline MOL000762 & palmidin A & C30H22O8 & 35.36 & 0.39 \\
\hline MOL000785 & palmatine & C21H24CINO5 & 64.6 & 0.13 \\
\hline MOL000098 & quercetin & C15H1007 & 46.43 & 0.38 \\
\hline MOL001458 & coptisine & C19H14CINO4 & 30.67 & 0.26 \\
\hline MOL002668 & worenine & C20H16NO4+ & 45.83 & 0.27 \\
\hline MOL008647 & moupinamide & C18H19NO4 & 86.71 & 0.33 \\
\hline The table lists the effective ingredients of Coptis chinensis obtained in the TCMSP database with OB \\
\hline$\geq 30$ and DL $\geq 0.18$ as the screening conditions. & & & \\
\hline TCMSP: TCM systems pharmacology database and analysis platform & & \\
\hline OB: Oral bioavailability & & & & \\
\hline DL: Drug-like & & & & \\
\hline COlleCtion & & & & \\
\hline
\end{tabular}

\section{Collection of targets for effective active ingredients of Coptis chinensis}

The component target model in the TCMSP database was searched, and there were 581 component targets for CC. Among them, hydrogenated berberine acted on 31 targets, berberine acted on 16 targets, berberine acted on 13 targets, and quercetin acted on 139 targets, which are the more effective targets. The Uniprot database was used to collect the gene names of the active targets and delete invalid and duplicate targets, leading to a total of 148 active targets of CC.

\section{Collection of Polycystic Ovary Syndrome -related genes}


The Genecards, OMIM, and PharmGkb disease databases were searched, and a total of 3,517 disease targets related to PCOS were collected. The R language was used to map the active targets of the active ingredients of $\mathrm{CC}$ to these disease targets. A Venn diagram showed that a total of 102 intersecting targets were obtained, which were defined as the "key targets" for CC to treat PCOS (Fig. 2). Among them, mitogen-activated protein kinase (MAPK1), insulin-like growth factor 2 (IGF2), and protein kinase B1 (Akt1), inter alia, were of significance in the occurrence and development of PCOS.

\section{Construction of a Common Target Interaction Network}

There were 102 nodes and 221 edges in the interaction network (Fig. 3). The frequency of occurrence of the 30 targets are shown in Fig. 4. Frequent protein interactions included v-jun avian sarcona virus 17 oncogene homolog (JUN), mitogen activated protein kinase (MAPK), interleukin (IL-6), recombinant human interleukin 8 (CXCL8), FOS, and a recombinant human interleukin 1 B (IL1B). Nodal proteins (indicating that the active components of $\mathrm{CC}$ had a high binding activity with them) can be used as potential targets for CC in treating PCOS.

\section{Construction of a drug-active, ingredient-disease-target, interaction network}

The components of $\mathrm{CC}$, the target on which the component acts, and the target of the disease were input into Cytoscape 3.7.1 to build a drug-active, component-disease-target, interaction network (Fig. 5). The network diagram contained 115 nodes, of which 102 nodes represented the target of the action; there were 259 edges indicating the relationship between nodes. The network showed that PTGS2, PTGS1, AR, and NCOA2 were linked to 9, 8, 8, and 7 components, respectively, and may serve as the central genes for CC to treat PCOS. Quercetin was linked to 91 genes, indicating that it may be the key active ingredient.

\section{Screening of core pathways for Coptis chinensis in treating polycystic ovary syndrome}

To determine whether the 102 identified genes were related to PCOS, a GO pathway enrichment analysis was performed to elucidate the relevant biological processes $(P<0.01)$. A bubble chart was drawn, using the OmicShare tools (www.omicshare.com/tools) (Fig. 4A). The GO analysis indicated that biological processes such as apoptosis, cytokine activation, oxidant antioxidant, and nitric oxide metabolism were mainly involved. CC may therefore treat PCOS by regulating multiple complex biological processes. Similarly, the KEGG pathway enrichment analysis of the above common targets was performed with the help of this network platform (Fig. 4B). Excluding extensive pathways, the top 20 signaling pathways are listed in Table 2. The 102 common targets were mainly distributed in multiple signaling pathways such as AGEs/RAGE, MAPK, PI3k/Akt, and TNF, which suggests that CC treats PCOS by acting on multiple pathways and that there is a gap between these pathways, indicating complex interactions. The size of the node indicates the number of enriched targets, and the color of the node from green to red indicates 
that the $P$ value changes from large to small. Therefore, the larger the red node, the higher the significance of the signal path.

In the Cytoscape 3.7.1 (https://cytoscape.org/) software, the ClueGo plug-in was used to perform GO term analysis on the common targets, and an interactive network and pie chart were obtained. The degree of correlation between the enriched biological processes and the number of genes included was described previously (Fig. 5A and 5B).

\section{Molecular docking results}

Molecular docking simplifies the study of the possible interaction modes of compounds and hub genes. The number of ligands bound to the receptor and its binding strength depend on the inhibition efficiency. Therefore, the interaction and binding mode of active ingredients (quercetin, berberine, berberrubine, canadine) and cellular inflammatory pathway related factors (MAPK1, CXCL8, IL-6, and IL-1 $\beta$ ) were studied through molecular docking. A score of $<5$ points indicates that the compound has excellent docking activity with the target. Docking scores of quercetin with MAPK1 (-6.894), CXCL8 (-5.185), IL-6 (-5.864), and IL-1 $\beta(-6.048)$ were the highest among the selected components. Quercetin forms covalent bonds with the ALA35, ASP111, ASP167, MET108, and LYS114 amino acid residues of MAPK1 (Fig. 6A, E); and forms covalent bonds with the LYS9 and GLU16 amino acid residues of CXCL8 (Fig. 6B, F). It forms covalent bonds with the LYS46, GLU42, SER107, SER108, and ASP160 amino acid residues of IL-6 (Fig. 6C, G), and forms covalent bonds with the LEU62, LEU67, and GLU64 amino acid residues of IL-1 $\beta$ (Fig. 6D, H), indicating that the combination is relatively stable. The molecular docking results between the remaining active ingredients and the target gene are shown in Table 3 and Additional File 1.

Table 3

Molecular docking score between active ingredients of $\mathrm{CC}$ and related targets

\begin{tabular}{|lllll|}
\hline Active ingredients & MAPK1 (6G54) & CXCL8 & IL-6 & IL-1 $\beta$ \\
& & $\mathbf{( 5 D 1 4 )}$ & $\mathbf{( 4 0 9 H )}$ & $\mathbf{( 4 G 6 M )}$ \\
\hline Quercetin(CID 5280343) & -6.894 & -5.185 & -5.864 & -6.048 \\
\hline Berberine (CID 2353) & -4.812 & -3.442 & -3.084 & -3.953 \\
\hline Canadine (CID 34458) & -4.316 & -3.581 & -4.900 & -4.261 \\
\hline Berberrubine(CID 72703) & -5.694 & -3.431 & -3.605 & -5.357 \\
\hline
\end{tabular}

\section{Discussion}

Polycystic ovary syndrome is an endocrine syndrome associated with ovulatory disorders and metabolic abnormalities. Since the main external characteristics of the disease are infrequent menstruation and prolonged infertility, in TCM, PCOS is mostly classified as "late menstruation", "less menstruation", "amenorrhea", "collapse", and "infertility". The etiology and pathogenesis of the disease (in TCM) involve kidney-Tiangui-Chongren-cycle axis dysfunction, phlegm dampness, and obstruction in the middle focus, 
with heat over time, resulting in kidney, liver, and spleen dysfunction. Coptis chinensis is a representative medicine for clearing away heat and dampness in TCM. At present, most clinical studies focus on the effect of the berberine extract component ("berberine"), but pharmacological studies have found that a Huang Lian (Chinese Herb) decoction is better than berberine monomers in improving insulin resistance, lowering blood sugar, and improving glucose and lipid metabolism disorders $[12,13]$. Due to the complex composition of TCM CC, many ingredients often participate in the process of exerting its effects, and the mechanisms of action are intertwined with each other. Therefore, it is necessary to conduct a comprehensive analysis on the relevant network pharmacology software platforms to interpret the mechanisms of action.

In this study, the main active ingredients of $\mathrm{CC}$ were found to include various organic components such as berberone, berberine, quercetin, canadine, and berberrubine. The core nodes in the "target" network suggest that these several active ingredients play a very important role in the treatment of PCOS. Other related studies have also verified that several components in CC improve follicular developmental disorders, ovulatory disorders, and lower fasting blood glucose and fasting insulin levels to reduce insulin resistance in women with PCOS [14, 15], concurring with the current study. A large, multicenter, randomized, double-blind, placebo-controlled trial of berberine in PCOS showed that berberine combined with the first-line, ovulation-promoting agent, letrozole, can improve ovulation rate and pregnancy [16]. In addition, berberine has the advantage of lowering the incidence of adverse gastrointestinal reactions and severe ovarian hyperstimulation syndrome, compared with letrozole.

Through analysis of drug and disease targets, it was found that quercetin has 139 targets, which are active ingredients with many targets. Quercetin is a natural flavonoid, which can inhibit the activity of aldose reductase, and has the effect of reducing the advanced glycation end products (AGEs) and insulin resistance. It has attracted the attention of researchers and clinicians [17]. The use of quercetin in the treatment of type 2 diabetes is becoming increasingly common. Treatment of diabetic rats with quercetin and metformin improved insulin sensitivity and increased liver glycogen levels, suggesting that the two substances act in a similar way [14]. In addition, quercetin can change the body composition of patients with obesity and dyslipidemia, thereby improving body metabolism and hormone levels [7]. Therefore, quercetin is also gradually applied to improve the treatment of patients with PCOS.

Analysis of the PPI network indicated that MAPK, JUN, IL-6, CXCL8, and FOS are at the core of the network and can be regarded as potential key targets for CC in treating PCOS. MAPK (a mitogen-activated protein kinase pathway) is involved in the regulation of oocyte maturation, and this effect is essential for normal follicular development and proper ovulation [18]. Once the MAPK pathway is blocked, mitotic cyclin D2 expression and the role of granulocyte proliferation will be impeded, resulting in a decrease in the number of granulocytes, which indirectly interferes with the development and maturation of follicles $[19,20]$. Experiments have confirmed that the high expression of IL-6 in PCOS may play a role in the pathogenesis of PCOS through the synergistic effect of insulin resistance and chronic inflammation [21]. The abnormally low expression of the FOS gene in granular cells may increase the androgen secretion of ovarian granular cells by reducing the inhibitory effect on the CYP17gene [22]. The results of molecular 
docking showed that the active ingredient, quercetin, with the most targets, had a higher degree of binding to MAPK, IL- $6, C X C L 8$, and IL-1 $\beta$, suggesting that the compound may play a therapeutic role by combining with these proteins.

ClueGO biological process enrichment analysis was performed on the key targets of the active components of $\mathrm{CC}$, which confirmed that $\mathrm{CC}$ has certain effects on biological processes such as apoptosis, cytokine activation, oxidant antioxidant, and nitric oxide metabolism. The process is reflected in the targets and related pathways involved in the treatment of PCOS by CC. In the KEGG enrichment analysis, CC was shown to regulate various PCOS pathogenesis-related signaling pathways including AGEs/RAGE, MAPK, PI3k/Akt, and TNF. Studies have shown that the high expression of AGEs plays a key role in the formation of PCOS. AGEs mainly bind to specific receptors (RAGE), and mediate a series of pathological responses through cellular signal transduction mechanisms [23].

Studies have found that after binding to AGEs, RAGE can inhibit the activation of phosphatidylinositol 3kinase (PI3K / Akt) and the expression of related genes in the course granule cells, so that the glucose transporter (GLUT4) can be transferred to the plasma membrane. Obstacles, inhibition of glycogen synthesis, inhibition of glycolysis, promotion of gluconeogenesis, inhibition of protein synthesis, and changes in gene expression promote elevated blood glucose, severe hyperinsulinemia, and IR. In the process of follicular development, oocytes find it difficult to synthesize glucose, and most of their energy comes from granulocyte glycolysis [24]. Therefore, AGEs/RAGE may reduce glucose intake by reducing the number of granulocyte membrane GLUT $₫ 4$ receptors and thereby reduce glucose intake, affecting oocyte growth [20]. In addition, up-regulated RAGE may reduce the sensitivity of granulocytes to follicle stimulating hormone (FSH) by inhibiting the MAPK/ERK $1 / 2$ pathway and promoting increased apoptosis of granulosa cells $[18,19]$. Therefore, for the treatment of PCOS, the active ingredients of CC can be selected to explore whether they plays a role through certain key targets and pathways. The research results reflect the characteristics of TCM with multiple components, multiple targets, and multiple approaches to treat the disease, and provide a reference for the treatment of PCOS. However, based on some limitations of network pharmacology and mechanical algorithms, it is necessary to further verify the mechanisms of action of CC on PCOS through clinical experiments.

\section{Conclusions}

Our study systematically explored the mechanisms of action of CC in PCOS treatment using a combination method that included network pharmacology and molecular docking. Through bioinformatics analysis, a total of 102 common targets related to CC and PCOS were identified. Network analysis identified MAPK1, JUN, IL-6, CXCL8/IL-8, FOS, IL1B, ESR1, and EGF as the hub targets and revealed that the cellular inflammatory pathway was a key pathway, which was enriched by multiple signaling pathways and multiple targets. Results from molecular docking suggested that the bioactive ingredients in CC exerted a therapeutic effect on PCOS by regulating the expression of MAPK1, IL-6, CXCL8 and IL1B to inhibit cell inflammation. 
This study provides a possible mode of action of CC for the treatment of PCOS. However, further studies, both in vitro and in vivo, are warranted to clarify the role and complicated mechanism of CC in the treatment of polycystic ovary syndrome.

\section{Abbreviations}

PCOS

Polycystic ovary syndrome

CC

Coptis chinensis

TCM

traditional Chinese medicine

TCMSP

TCM systems pharmacology

PPI

protein-protein interaction

KEGG

Kyoto Encyclopedia of Genes and Genomes

MAPK1

mitogen-activated protein kinase

IGF2

insulin-like growth factor 2

Akt1

protein kinase B1

IL-6

interleukin

CXCL8

recombinant human interleukin 8

IL1B

recombinant human interleukin $1 \mathrm{~B}$

AGEs

advanced glycation end products

$\mathrm{FSH}$

follicle stimulating hormone

\section{Declarations}

Ethics approval and consent to participate: Not applicable

Consent for publication: Not applicable 
Availability of data and materials

All data generated or analysed during this study are included in this published article and its additional files.

\section{Competing interests}

The authors declare that there are no competing interests associated with the manuscript.

\section{Funding}

This work was supported by grants from the Natural Science Foundation of Zhejiang Province, China [Grant numbers LQ19H270005هGF20H270004] and Hangzhou Health Science and Technology Plan Project [Grant numbers 2018A38, 002019011, 002019008].

\section{Authors' contributions:}

JM and HT conceived and designed the study. XW designed the molecular docking. MY and KM made language changes to the manuscript. All authors read and approved the final manuscript.

Acknowledgements: We would like to thank Editage for English language editing.

\section{References}

1. Hadjiconstantinou M, Mani H, Patel N, Levy M, Davies M, Khunti K, et al. Understanding and supporting women with polycystic ovary syndrome: a qualitative study in an ethnically diverse UK sample. Endocr Connect. 2017;6:323-30.

2. Busiah K, Colmenares A, Bidet M, Tubiana-Rufi N, Levy-Marchal C, Delcroix C, et al. High prevalence of polycystic ovary syndrome in Type 1 Diabetes Mellitus adolescents: Is there a difference depending on the NIH and Rotterdam Criteria. Horm Res Paediatr. 2017;87:333-41.

3. Ndefo UA, Eaton A, Green MR. Polycystic ovary syndrome: a review of treatment options with a focus on pharmacological approaches. P T. 2013;38:336-55.

4. Hwang KR, Choi YM, Kim JJ, Chae SJ, Park KE, Jeon HW, et al. Effects of insulin-sensitizing agents and insulin resistance in women with polycystic ovary syndrome. Clin Exp Reprod Med. 2013;40:100-5.

5. Li Cai-hong. Zhou Ke-yuan. Study advances on the effects and mechanisms of active constituents of Coptidis Rhizoma. Lishizhen Med Materia Medica Res. 2010;21:466-8.

6. $10.1155 / 2015 / 905749$

Bing P, Lin-Hua Z, Qiang Z, Zhao TY, Wang H, Gu CJ, et al. Application of berberine on treating type 2 diabetes mellitus. Int J Endocrinol. 2015; 2015:905749. doi:10.1155/2015/905749.

7. Wei W, Zhao H, Wang A, Sui M, Liang K, Deng H, et al. A clinical study on the short-term effect of berberine in comparison to metformin on the metabolic characteristics of women with polycystic 
ovary syndrome. Eur J Endocrinol. 2012;166:99-105.

8. Li L, Li C, Pan P, Chen X, Wu X, Ng EH, et al. A single arm pilot study of effects of berberine on the menstrual pattern, ovulation rate, hormonal and metabolic profiles in anovulatory Chinese women with polycystic ovary syndrome. PLoS ONE. 2015;10:e0144072.

9. Geng Q, Guo J, Zhang J, Wang F. A meta-analysis of the Chinese medicine Bushenhuoxue for treatment of ollgospermia and astheuospermia. Chin J Fam Plan. 2012;20:303-6.

10. Dong B, Wang RH, Liu LM. Review on common methods and technologies of quantitative composition-activity relationship research of TCM. Chinese J Info Trad Chinese Med. 2017;24:12630.

11. Hopkins AL. Network pharmacology. Nat Biotechnol. 2007;25:1110-1.

12. Fu Y, Hu BR, Tang Q, Fu Q, Zhang QY, Xiang JZ. Effect of jatrorrhizine, berberine, Huanglian decoction and compound-mimic prescription on blood glucose in mice. Chinese Trad Herb D. 2005;36:548-51.

13. Lai X, Zhang Y, Zheng H, Deng C, Fan G, Zhou L, et al. Study on effective substances of San Huang preparation and its single herbs by serum pharmacochemistry. World Sci Tech- Modernization Trad Chinese Med. 2010;12:666-70.

14. An Y, Sun Z, Zhang Y, Liu B, Guan Y, Lu M. The use of berberine for women with polycystic ovary syndrome undergoing IVF treatment. CLIN ENDOCRINOL (Oxf). 2014;80:425-31.

15. Li Y, Kuang H, Shen W, Ma H, Zhang Y, Stener-Victorin E, et al. Letrozole, berberine, or their combination for anovulatory infertility in women with polycystic ovary syndrome: study design of a double-blind randomised controlled trial. BMJ Open. 2013;3:e003934.

16. Wu XK, Wang Y, Liu JP, Hou LH, Gao YQ, Du SM, et al. Letrozole, berberine, or a combination for infertility in Chinese women with polycystic ovary syndrome: a multicentre, randomised, double-blind, placebo-controlled trial. Lancet. 2015. doi:10.1016/S0140-6736(15)00651-0.

17. Rezvan N, Moini A, Janani L, Mohammad K, Saedisomeolia A, Nourbakhsh M, et al. Effects of quercetin on adiponectin-mediated insulin sensitivity in polycystic ovary syndrome: a randomized placebo-controlled double-blind clinical trial. Horm Metab Res. 2017;49:115-21.

18. Su YQ, Wigglesworth K, Pendola FL, O'Brien MJ, Eppig JJ. Mitogen-activated protein kinase activity in cumulus cells is essential for gonadotropin-induced oocyte meiotic resumption and cumulus expansion in the mouse. Endocrinology. 2002;143:2221-32.

19. Kandaraki EA, Chatzigeorgiou A, Papageorgiou E, Piperi C, Adamopoulos C, Papavassiliou AG, et al. Advanced glycation end products interfere in luteinizing hormone and follicle stimulating hormone signaling in human granulosa KGN cells. Exp Biol Med (Maywood). 2018;243:29-33.

20. Kayampilly PP, Menon KM. Follicle-stimulating hormone inhibits adenosine 5'-monophosphateactivated protein kinase activation and promotes cell proliferation of primary granulosa cells in culture through an Akt-dependent pathway. Endocrinology. 2009;150:929-35.

21. Wang J, Zhu L, Hu K, Tang Y, Zeng X, Liu J, et al. Effects of metformin treatment on serum levels of C-reactive protein and interleukin- 6 in women with polycystic ovary syndrome: a meta-analysis: $A$ PRISMA-compliant article. Medicine. 2017;96:e8183. 
22. Sang M, Li J, Zhang Y, Wu X. The mechanism of c-fos gene regulation of 17-alpha hydroxylase (CYP17) expression and testosterone production in ovarian granulosa cells from polycystic ovary

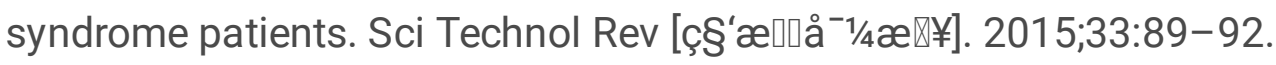

23. Stensen MH, Tanbo T, Storeng R, Fedorcsak P. Advanced glycation end products and their receptor contribute to ovarian ageing. Hum Reprod. 2014;29:125-34.

24. Chang HM, Klausen C, Leung PC. Antimüllerian hormone inhibits follicle-stimulating hormoneinduced adenylyl cyclase activation, aromatase expression, and estradiol production in human granulosa-lutein cells. Fertil Steril. 2013;100:585 - 92.e1.

\section{Tables}

Due to technical limitations, table 2 is only available as a download in the Supplemental Files section.

\section{Figures}




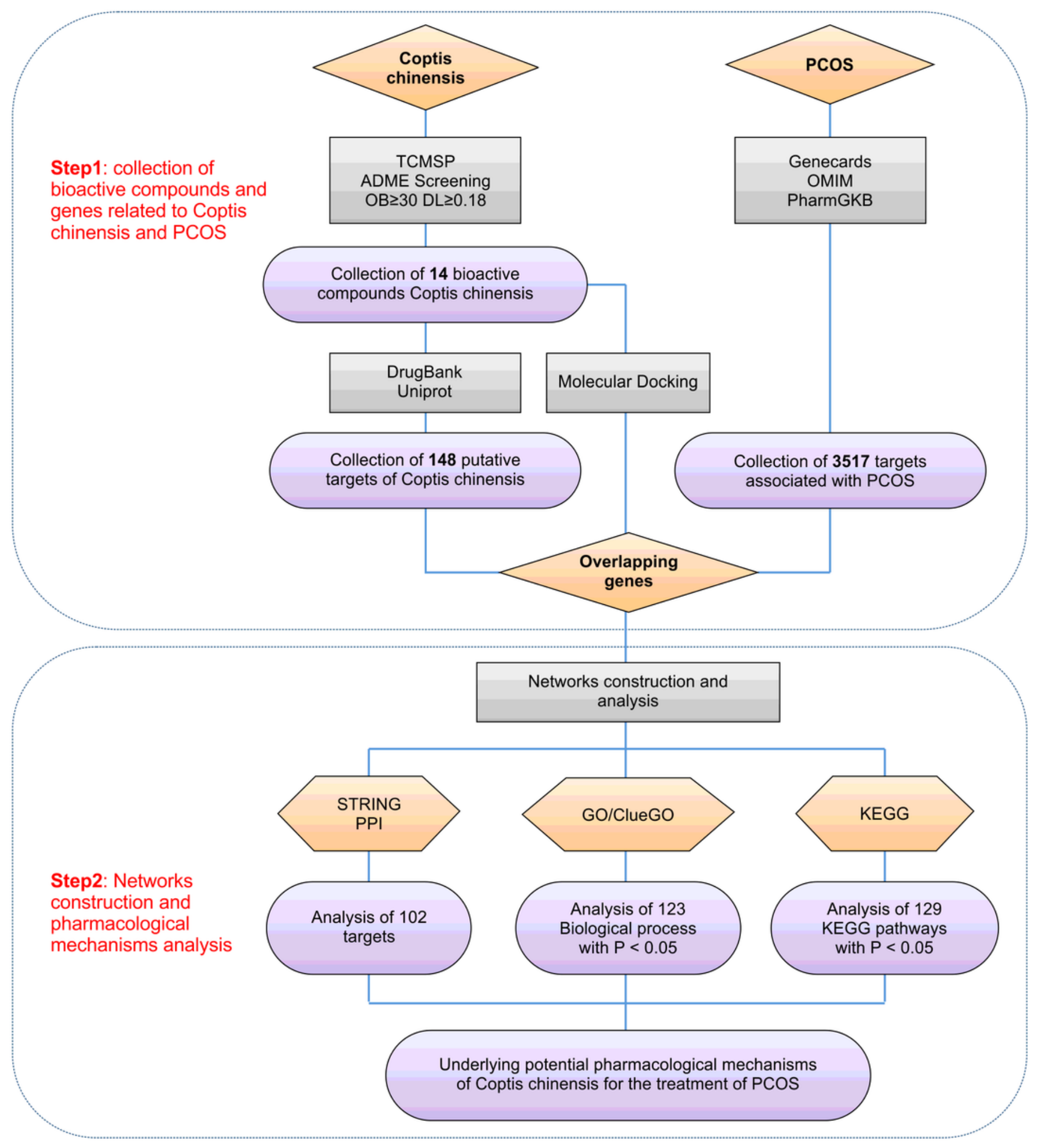

\section{Figure 1}

Flow chart depicting the network pharmacology approach used in this study. 

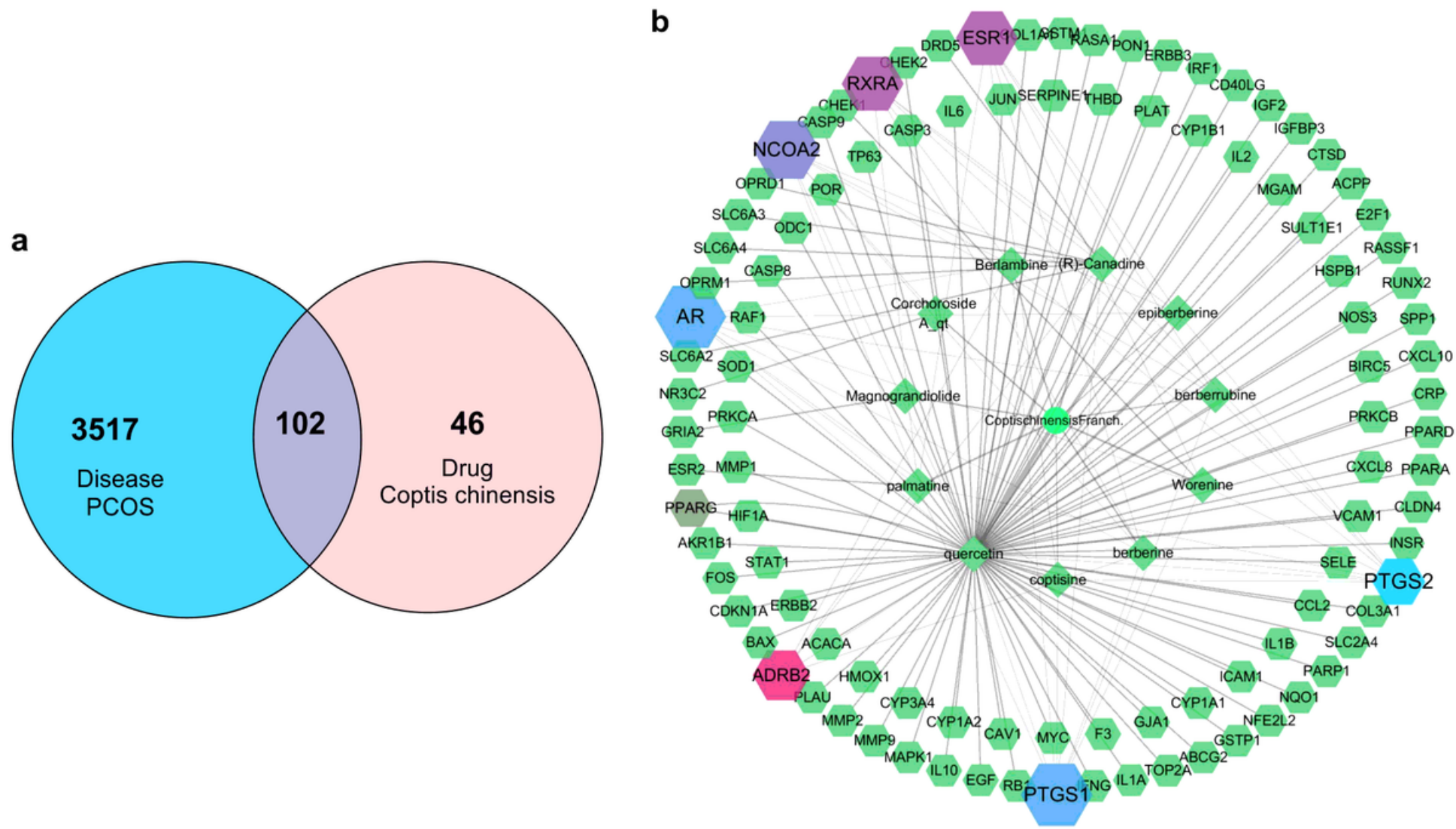

\section{Figure 2}

The Venn diagram of overlapping genes and Network of Drug-Ingredients-Genetics. (A) The 102 overlapping genes between the drug and disease. (B) Network of Drug-Ingredients-Genes. The 102 hexagonal nodes represent the overlapping genes between the drug and disease. The 14 diamondshaped nodes represent the active ingredients in CC. The edges in the figure denote the nodes that can interact with each other. 
a

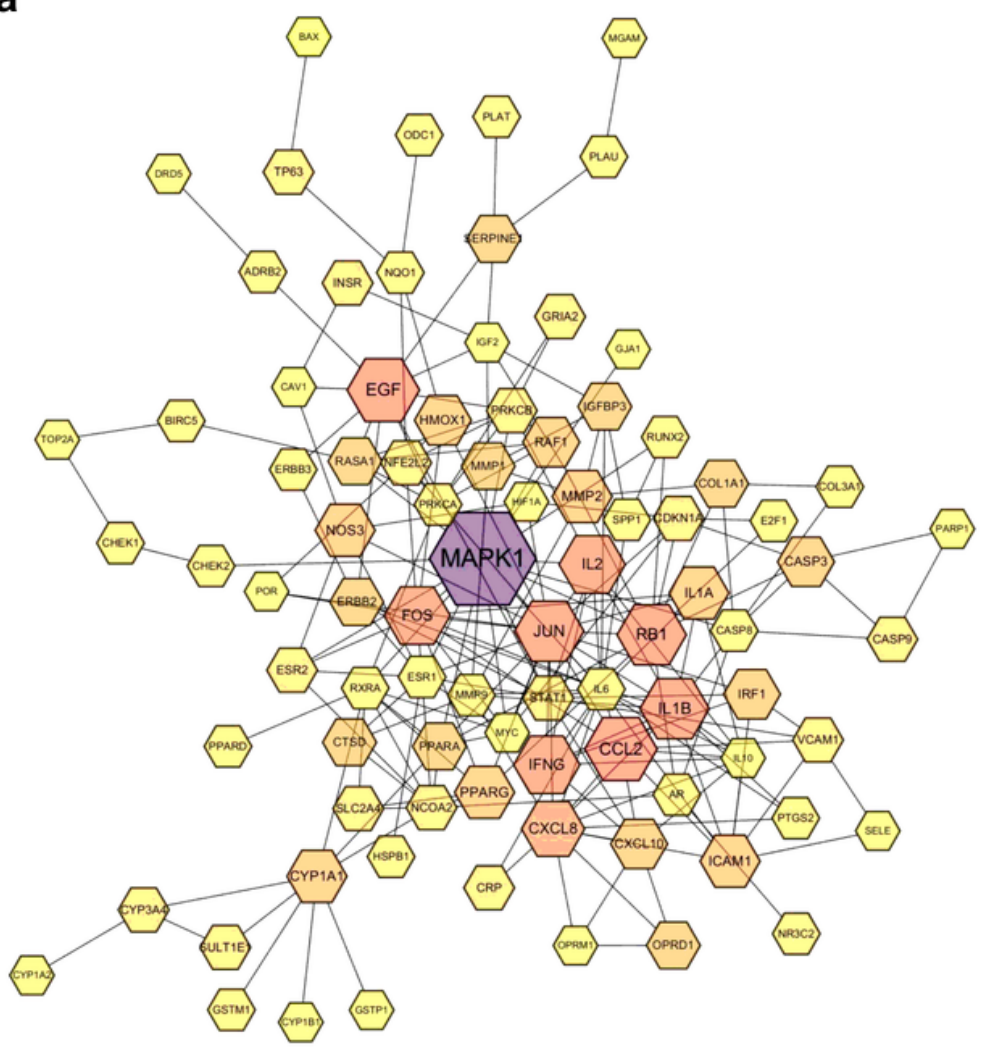

b

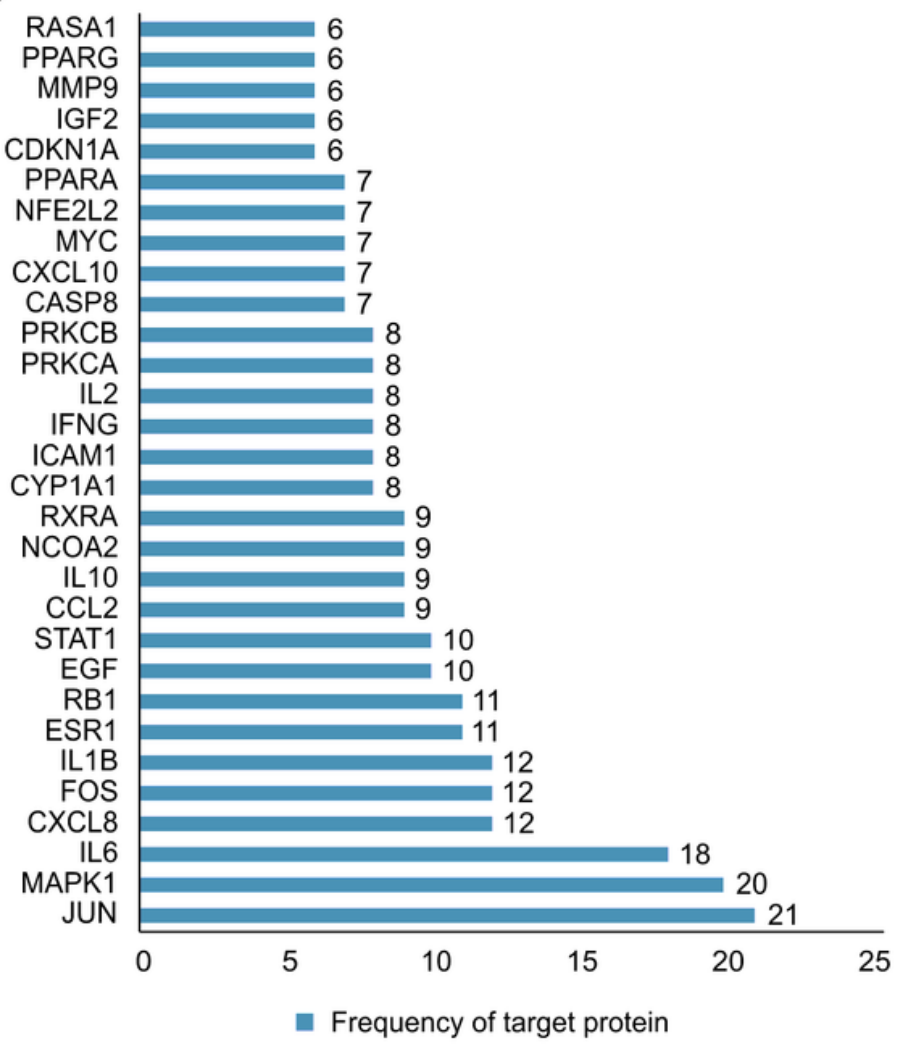

Figure 3

The protein-protein interaction (PPI) network. (A) Target protein-protein interaction network (PPI) of biologically active ingredients related to the treatment of polycystic ovary syndrome in CC. Each node represents a related target gene. A greater degree of target PPI is represented by darker colors and larger nodes, and edges with higher merge scores are denoted by darker and thicker lines. (B) Bar graph of the frequency of PPI network target proteins. The x-axis represents the number of target proteins connected to neighboring proteins, whereas the $y$-axis represents the top 30 target proteins with higher interaction frequencies.

a

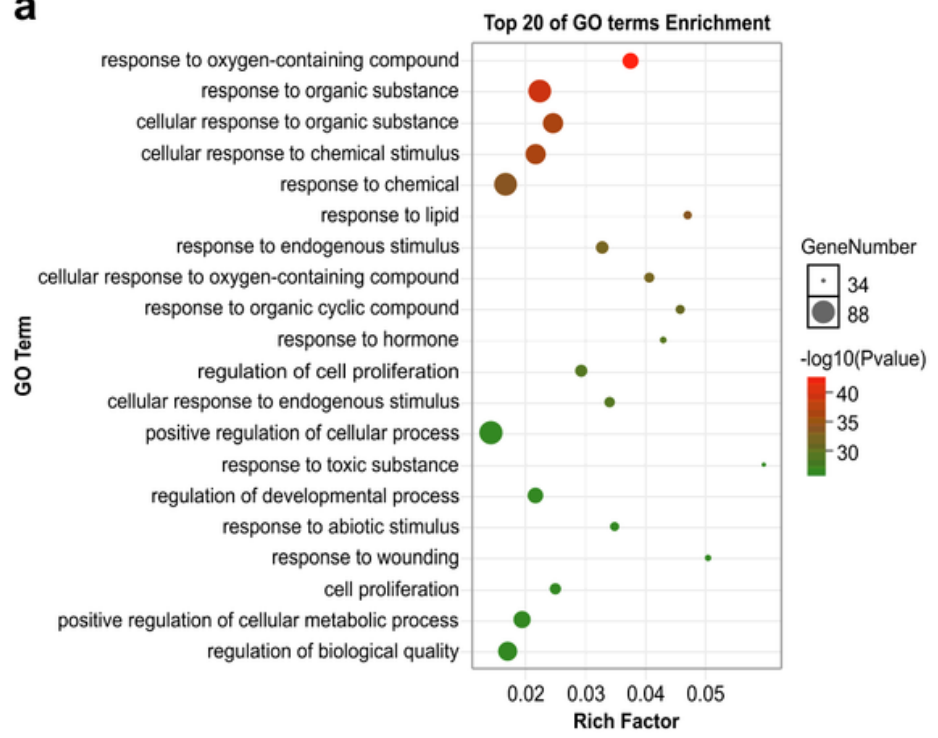

b

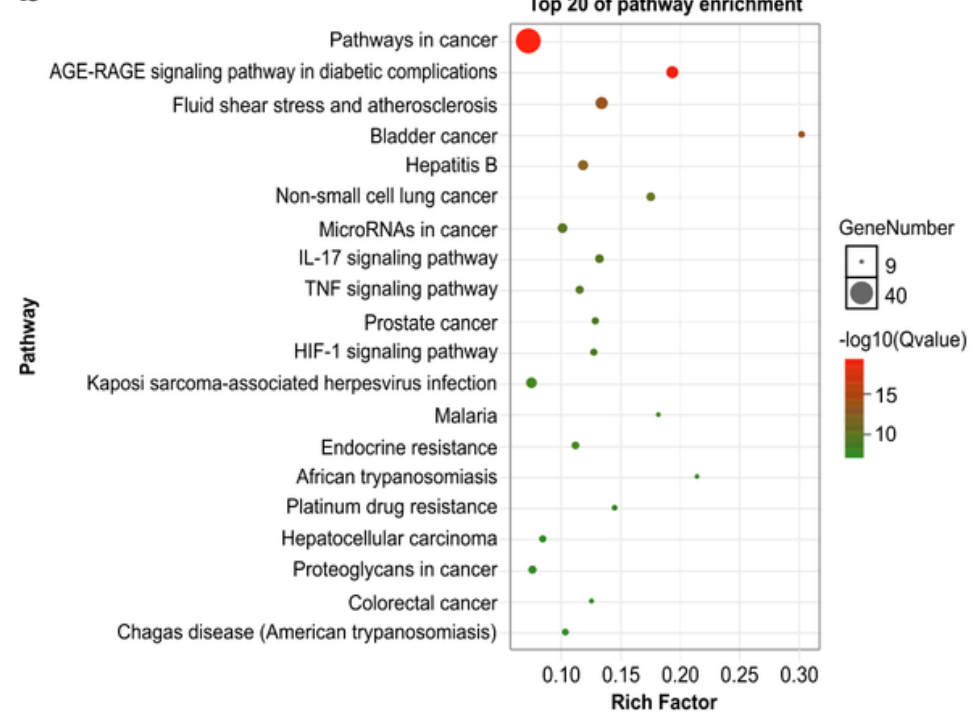




\section{Figure 4}

Enrichment analysis of gene ontology (GO) biological processes and KEGG signaling pathway enrichment analyses. (A) GO tool-based analysis of 102 genes related to polycystic ovary syndrome. The $x$-axis represents the enrichment factor of the target gene, and the $y$-axis represents the "biological process" category where the target gene is enriched in $G O(P<0.01)$. The size of the bubble area represents the number of genes belonging to the $\mathrm{GO}$ item in the target gene. The color of the bubble indicates the concentration, and a darker color indicates a higher importance. (B) KEGG signal pathway enrichment analysis. The $x$-axis represents the enrichment factor of the target gene, whereas the $y$-axis represents the main signal pathway $(P<0.01)$; the size of the bubble in the figure represents the number of genes belonging to the pathway in the target gene. The color of the bubble indicates the importance of enrichment; a red color indicates a higher importance. 


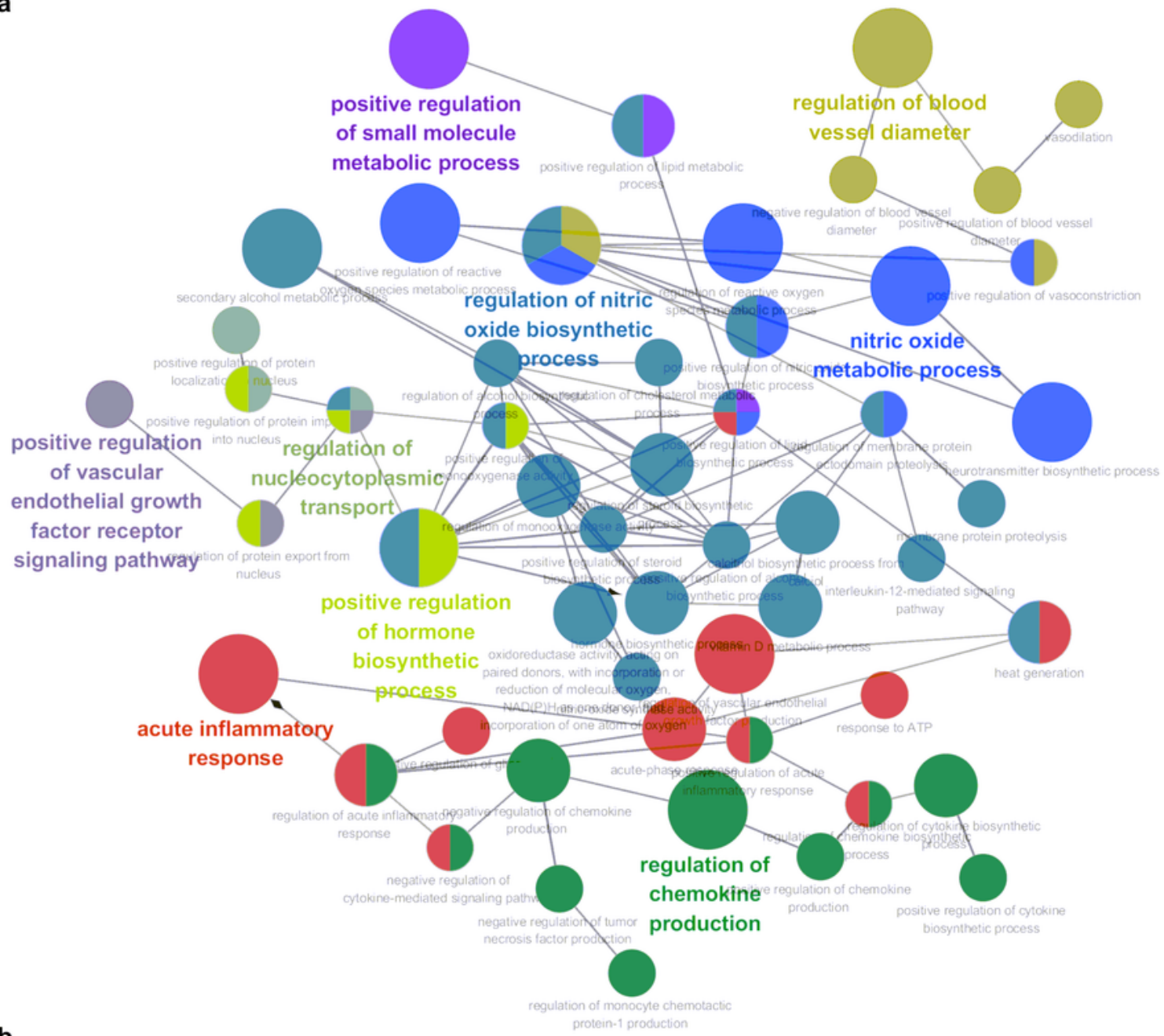

b

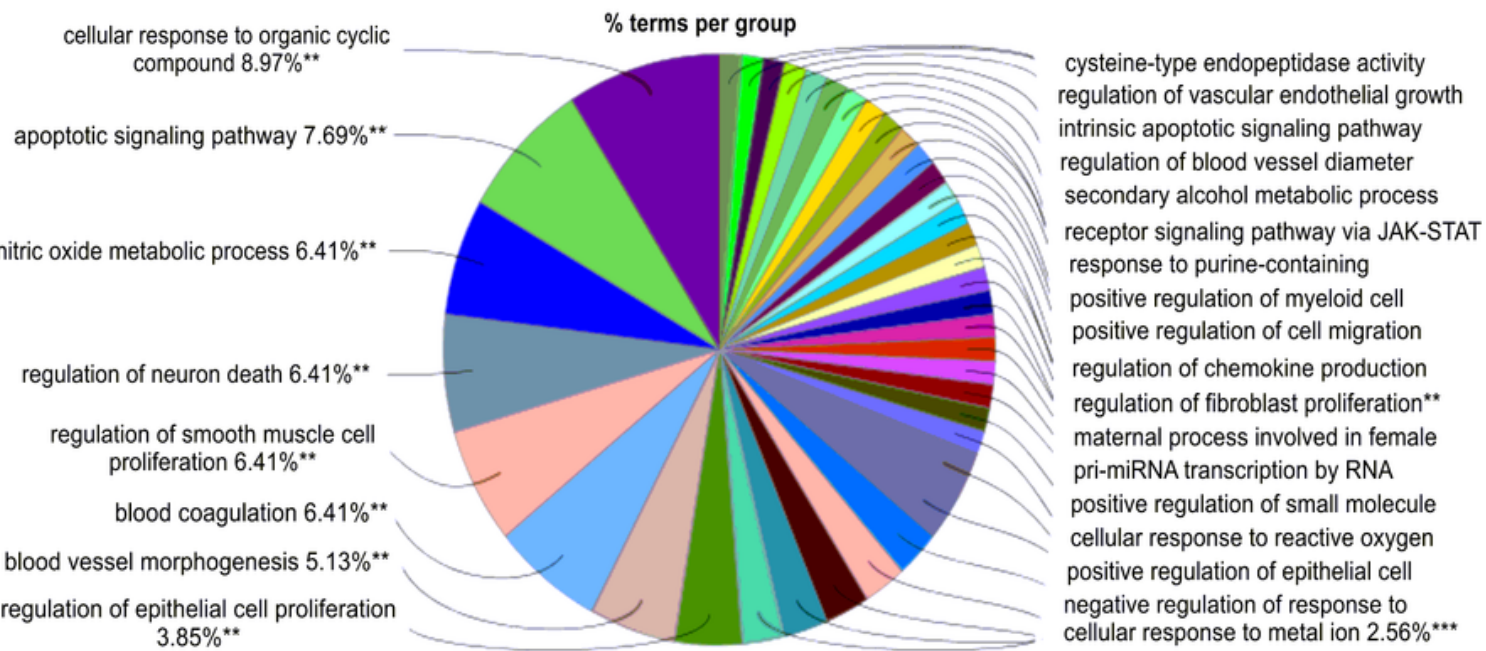

Figure 5

Biological function classification of 102 genes related to polycystic ovary syndrome using the Cluego tool. (A) The Cluego tool analyzed 102 genes related to polycystic ovary syndrome. Different node colors represent the biological process of enrichment. The size of the node indicates the degree of enrichment. The larger the node, the higher the degree of enrichment. The connections are represented by a line. (B) 
Enrichment analysis of the gene ontology (GO) terms. The area of the pie chart represents the proportion of the target genes enriched in different biological processes.

a

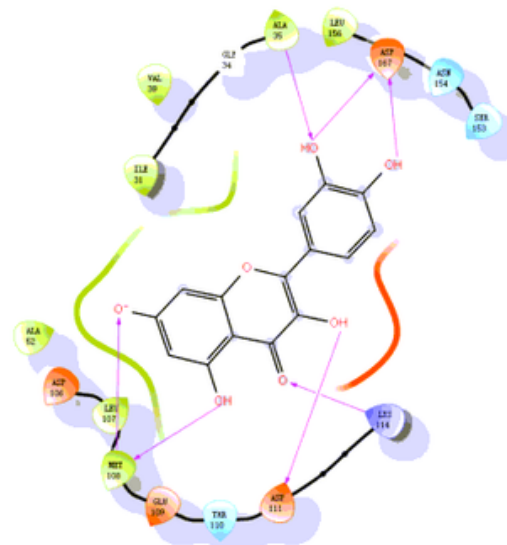

b

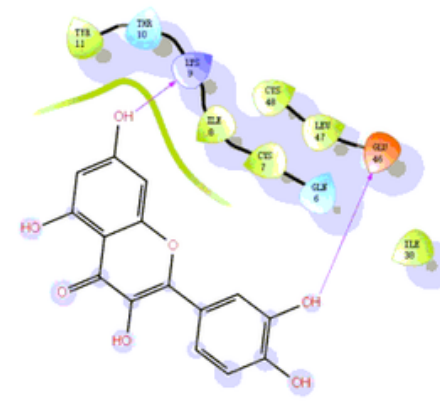

c

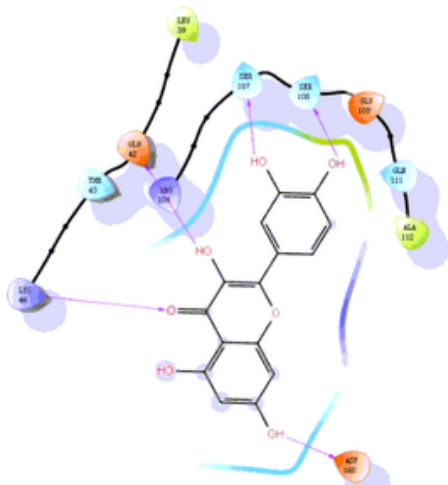

d

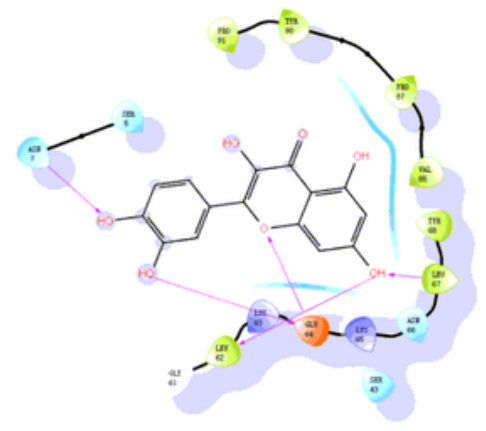

e

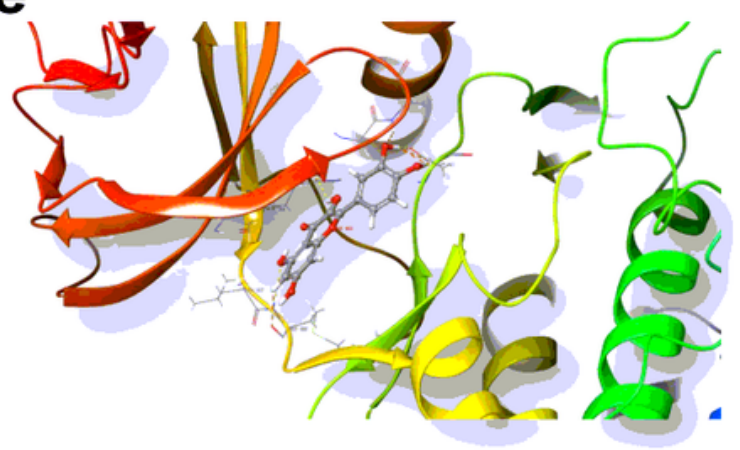

$\mathbf{f}$

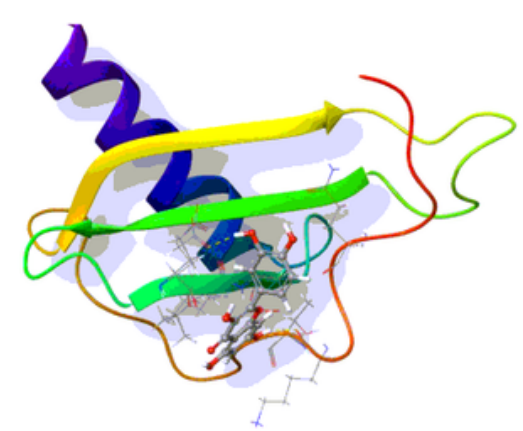

g

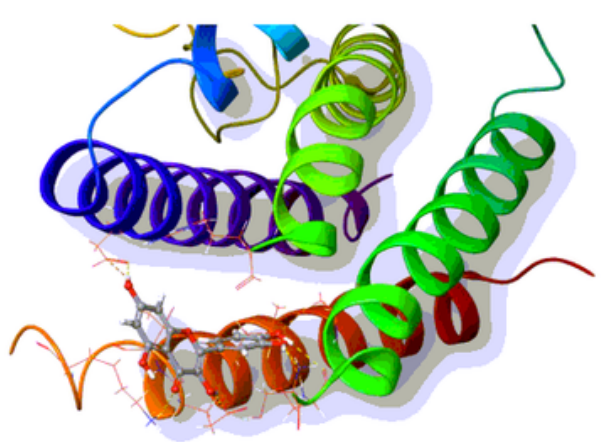

h

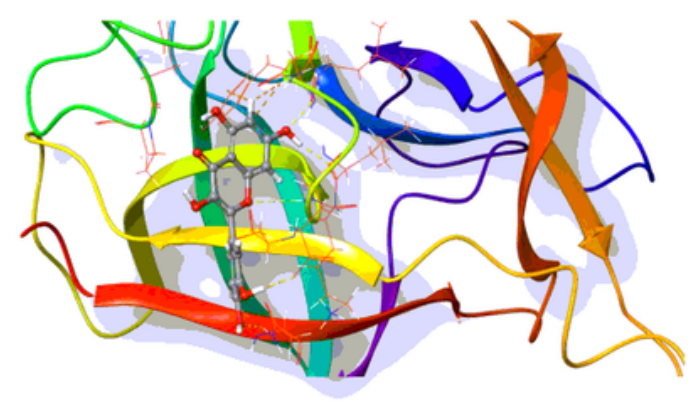

Figure 6

Molecular docking between quercetin and related targets. A and $E$ represent the two-dimensional and three-dimensional results of virtual molecular docking of MAPK1 and quercetin, respectively. $\mathrm{B}$ and $\mathrm{F}$ represent the two-dimensional and three-dimensional results of virtual molecular docking of CLCX8 and 
quercetin, respectively. $\mathrm{C}$ and $\mathrm{G}$ represent the two-dimensional and three-dimensional results of virtual molecular docking of IL- 6 and quercetin, respectively. $\mathrm{D}$ and $\mathrm{H}$ represent the two-dimensional and threedimensional results of virtual molecular docking of IL-1 $\beta$ and quercetin, respectively.

\section{Supplementary Files}

This is a list of supplementary files associated with this preprint. Click to download.

- Table2.docx

- AdditionalFile1.tif 